\title{
Resensitization to Nivolumab after Intratumoral Chemotherapy in Recurrent Head and Neck Squamous Cell Cancer: A Report of 2 Cases
}

\author{
Aline Houessinon ${ }^{a, c}$ Aurélie Moreira ${ }^{a}$ Jérèmie Bettoni ${ }^{b, c}$ \\ Marine Schoonaker ${ }^{c, d} \quad$ Chloé Sauzay ${ }^{c, d}$ Antoine Galmiche ${ }^{c, d}$ \\ Alexandre Coutte ${ }^{e} \quad$ Aurélie Biet $^{f} \quad$ Claude Krzisch $^{e}$ Bruno Chauffert ${ }^{a, c}$ \\ ${ }^{a}$ Department of Medical Oncology, University Hospital, Amiens, France; bepartment of \\ Maxillo-Facial Surgery, University Hospital, Amiens, France; ' $E A 7516$ CHIMERE, Picardie Jules \\ Verne University, Amiens, France; 'Laboratory of Biochemistry, University Hospital, Amiens,

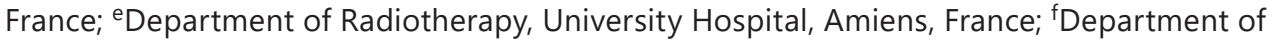 \\ ENT and Cervico-Facial Surgery, University Hospital, Amiens, France
}

Keywords

Head and neck cancer - Nivolumab - Intratumoral chemotherapy · Abscopal-like effect

\begin{abstract}
The survival of patients with head and neck squamous cancer with locoregional recurrence is short if salvage surgery or radiation cannot be performed. Systemic chemotherapy based on platinum salts and cetuximab produces only partial and transient responses. Immune checkpoint inhibitors (i.e., nivolumab) lead to a low complete response rate of only about $10 \%$, but in some cases the effects can be long-lasting. Intratumoral chemotherapy (ITC) has been proposed for patients with local recurrence of head and neck squamous cell carcinoma with an objective response rate of $27-50 \%$. However, it often leads to peritumoral tissue necrosis, and the duration of local control is limited. Here, we present 2 patients with head and neck squamous cell cancer whose local recurrences were refractory to intravenous chemotherapy and nivolumab. ITC using nonnecrotizing molecules, associated with nivolumab, led to complete stable local and distant response. ITC seems to trigger tumor resensitization to previously ineffective immunotherapy. This combination deserves an evaluation in the framework of a prospective trial.




\section{Introduction}

In Europe, head and neck squamous cell cancers (HNSCC) represent $4 \%$ of all malignancies [1], occurring mainly in men. Prognosis remains poor with a $60 \%$ locoregional failure and 30\% metastatic relapse after local treatment [2]. Therapeutic options in recurrent or metastatic HNSCC are limited to platinum-based chemotherapy and cetuximab (monoclonal antibody directed against the epidermal growth factor receptor), with a response rate of $36 \%$ and a few long-term complete responses [3, 4]. Anti-PD-1 immune checkpoint inhibitors, such as nivolumab, have recently been approved for the treatment of recurrent and/or metastatic HNSCC. Complete response rate remains low, approaching 10\% [5]. In Europe, nivolumab is currently the gold standard treatment for recurrent HNSCC that are refractory to chemotherapy-based regimens.

Intratumoral chemotherapy (ITC) has been proposed for patients with local recurrence of HNSCC, for whom traditional therapeutic approaches have failed. The objective response rate varies from 27 to 50\% [6]. Despite these encouraging results, this technique presents some limitations: the drug distribution in tumors is often nonhomogeneous, the long-term local control rate is low, and side effects, such as pain and peritumoral tissue necrosis, can occur.

We report 2 patients with long-term complete remission using ITC combined with intravenous (IV) nivolumab in recurrent HNSCC that were previously refractory to immunotherapy. These encouraging results could perhaps be explained by drug-induced modulation of PDL-1 expression in HNSCC cells.

\section{Case Reports}

Case 1

A 65-year-old former smoker, with a medical history of atrial fibrillation treated with fluindione and propranolol and diabetes treated with sitagliptin, presented isolated lymphadenopathies in right cervical nodes, levels II and III, in April 2015. The endoscopic examination of the airways and the extension assessment were considered normal (cTxN2M0). He underwent a right cervical dissection. The histological analysis was consistent with squamous cell carcinoma in 2 of the 16 nodes that were removed. Adjuvant radiotherapy delivering 66 Gy in 33 fractions was then carried out. In December 2015, a supraglottic carcinoma was discovered with left level III cervical lymphadenopathy. The patient underwent a left cervical dissection resulting in 9 pathological lymph nodes among the 23 removed, followed by radiotherapy on both the laryngeal tumor (66 Gy, 33 fractions) and the lymph nodes with concomitant cetuximab. In October 2016, several skin nodules appeared at the level of the right cervical dissection scar. The patient received nivolumab (3 mg/kg every 15 days) from January 2017 to March 2017, but clinical progression led to discontinuation. He then received docetaxel, carboplatin, and cetuximab from April 2017 to December 2017, which were stopped owing to local progression after stabilization. From January 2018 to May 2018, gemcitabine associated with methotrexate were administered: initially, a partial local response was obtained, but progression occurred locally and at a distance with left iliac and axillary metastatic nodes. From June to July 2018, nivolumab was re-introduced, but local progression appeared after 2 injections. The patient then received ITC with cisplatin $20 \mathrm{mg} / \mathrm{L}$ and gemcitabine $200 \mathrm{mg} / \mathrm{L}$ by 24-h infusion using 1 catheter. The absence of mixture precipitation was verified visually. ITC was performed on July 17, 2018, July 31, 2018, and September 4, 2018. A complete local response was obtained (Fig. 1A-C). In November 2018, 18-fluorodesoxyglucose positron emission tomography (18-FDG-PET) scan showed hyperfixation of a

\section{Karger'}




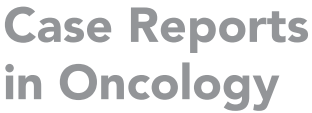

Case Reports

in Oncology

\begin{tabular}{l|l}
\hline Case Rep Oncol 2020;13:835-842 \\
\hline DOI: 10.1159/000507986 & $\begin{array}{l}\text { @ 2020 The Author(s). Published by S. Karger AG, Basel } \\
\text { www.karger.com/cro }\end{array}$ \\
\hline
\end{tabular}

Houessinon et al.: Resensitization to Nivolumab after Intratumoral Chemotherapy

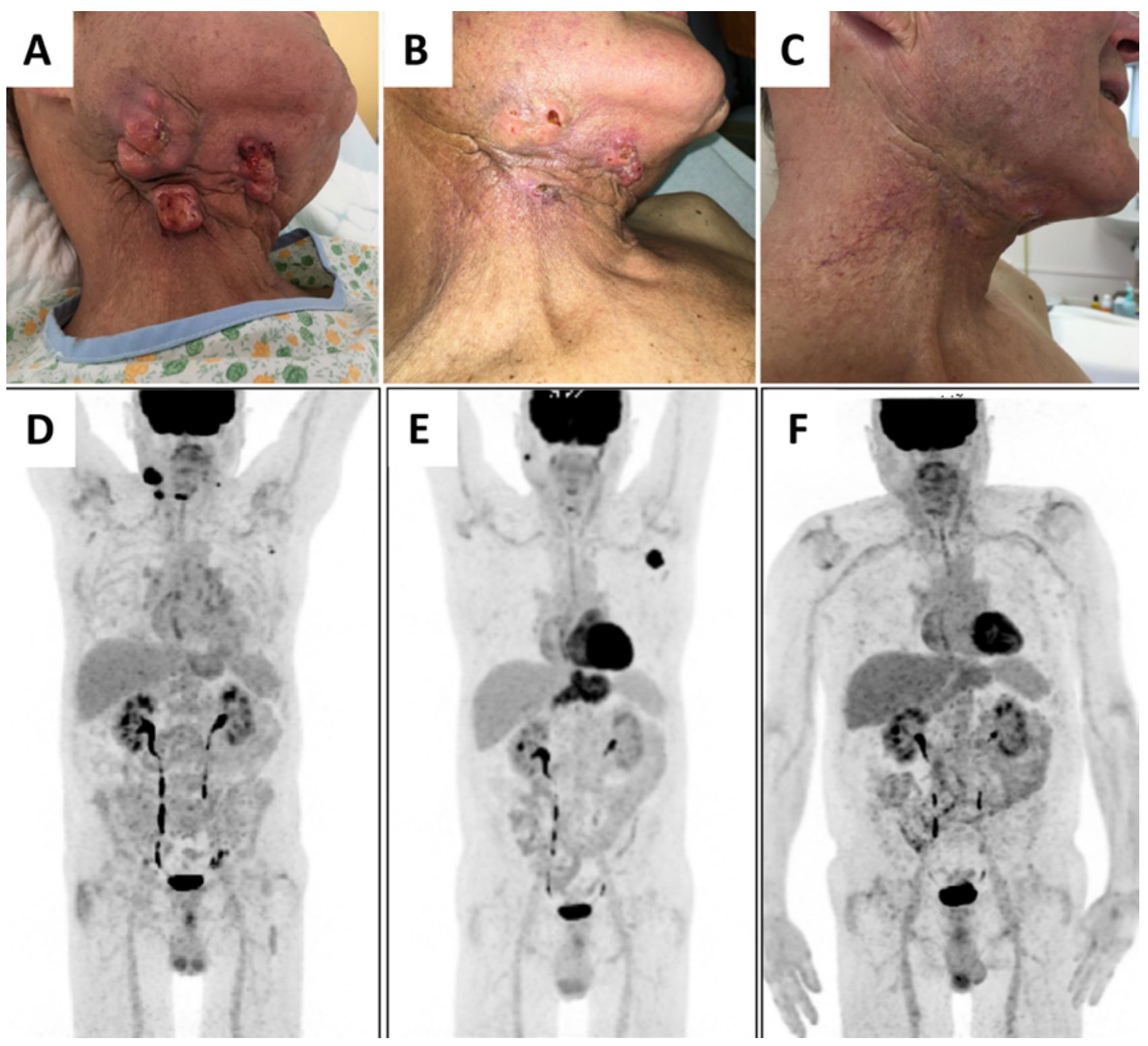

Fig. 1. A July 2018: Recurrent cervical tumor before starting ITC with cisplatin $20 \mathrm{mg} / \mathrm{L}$ and gemcitabine 200 mg/L by 24-h infusion. B September 2018: Significant regression before the third intratumoral injection. C February 2020: Sustained local complete remission. D May 2018: 18-FDG-PET scan before ITC. E November 2018: Local metabolic response but axillary node progression. F December 2019: Normalization of 18-FDG-PET scan after restarting IV nivolumab with sustained response.

3-cm left mobile axillary lymphadenopathy (Fig. 1D). Nivolumab was resumed on January 17, 2019 (240 mg every 15 days). The left axillary lymphadenopathy completely disappeared after the first injection of nivolumab. The 18-FDG-PET scan on August 5, 2019, and then on December 5, 2019, showed a complete metabolic response (Fig. 1E, F). There was no skin recurrence. Nivolumab was discontinued in December 2019. The patient was in complete remission on February 26, 2020, 20 months after the first ITC.

\section{Case 2}

A 64-year-old former smoker with a history of alcohol-induced cirrhosis. The patient was weaned off alcohol. He presented with a squamous carcinoma of the base of the tongue in February 2016 (cT4aN3M0). Radiotherapy on both the tumor and the lymph nodes (69 Gy, 30 fractions) was given with concomitant carboplatin and 5-fluorouracil chemotherapy. He presented a recurrence with a right retro-mandibular lymphadenopathy treated with docetaxel, 


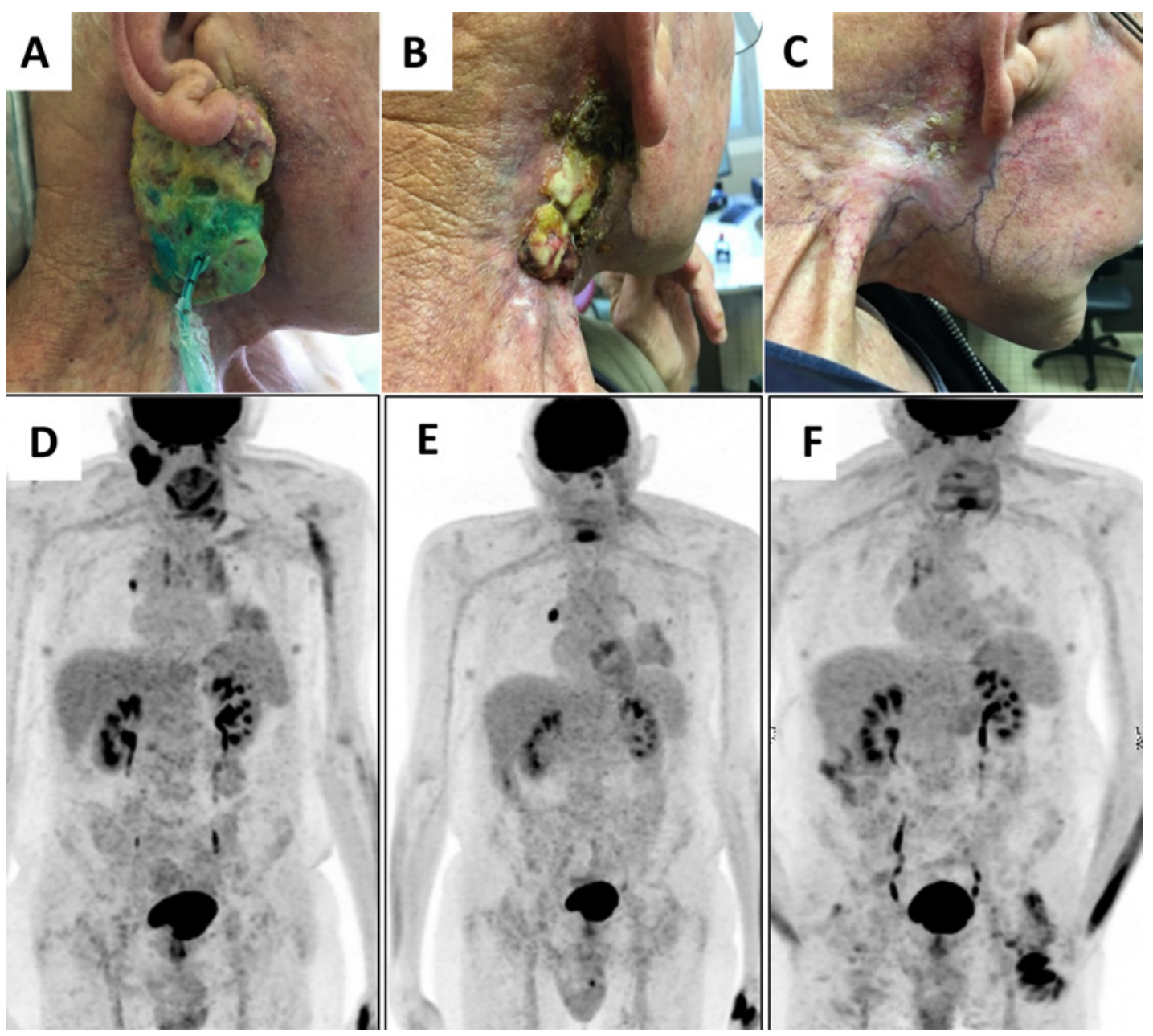

Fig. 2. A January 2019: Recurrent retromandibular tumor before starting ITC with carboplatin $20 \mathrm{mg} / \mathrm{L}$, gemcitabine $200 \mathrm{mg} / \mathrm{L}$, and paclitaxel $20 \mathrm{mg} / \mathrm{L}$ by 24-h infusion. Note the partial distribution of the drug mixture dyed by Patent Blue. B April 2019: Slow tumor regression with dry necrosis after 2 rounds of ITC. C March 2020: Complete local response 13 months after ITC and restarting IV nivolumab. D FDG-PET scan before ITC. Note the tumor hyperfixation and a right hilar node fixation. E October 2019: Disappearance of the 18-FDG fixation in the retromandibular area after ITC, but increased FDG fixation in the right hilar area. F March 2020: Normalization of 18-FDG-PET scan after hypofractionated radiotherapy on the right hilar node.

carboplatin, and cetuximab from January to July 2018. A transient partial response was achieved. Nivolumab (240 mg/15 days) was administered from August to October 2018 with no efficacy. Two courses of docetaxel with carboplatin also had no effect. The patient then received intratumoral carboplatin $20 \mathrm{mg} / \mathrm{L}$, gemcitabine $200 \mathrm{mg} / \mathrm{L}$, and paclitaxel $20 \mathrm{mg} / \mathrm{L}$ by $24 \mathrm{~h}$-infusions on January 17, 2019, and February 8, 2019. The absence of mixture precipitation was verified visually. Patent Blue dye was added during the first infusion to visualize the distribution of the liquid (Fig. 2A). One intratumoral catheter was used at each ITC administration. Despite only a partial perfusion of the tumor, a partial and then a complete local response was obtained (Fig. 2B, C). Nivolumab was re-administered from February 2019 to date (March 2020). Since a hyperfixing lymphadenopathy persisted at the right hilar level on the 18-FDG-PET scan in October 2019 (Fig. 2E), irradiation was carried out (30 Gy, 5 fractions). In March 2020, 14 months after the first ITC, the 18-FDG-PET scan no longer showed significant fixation. 


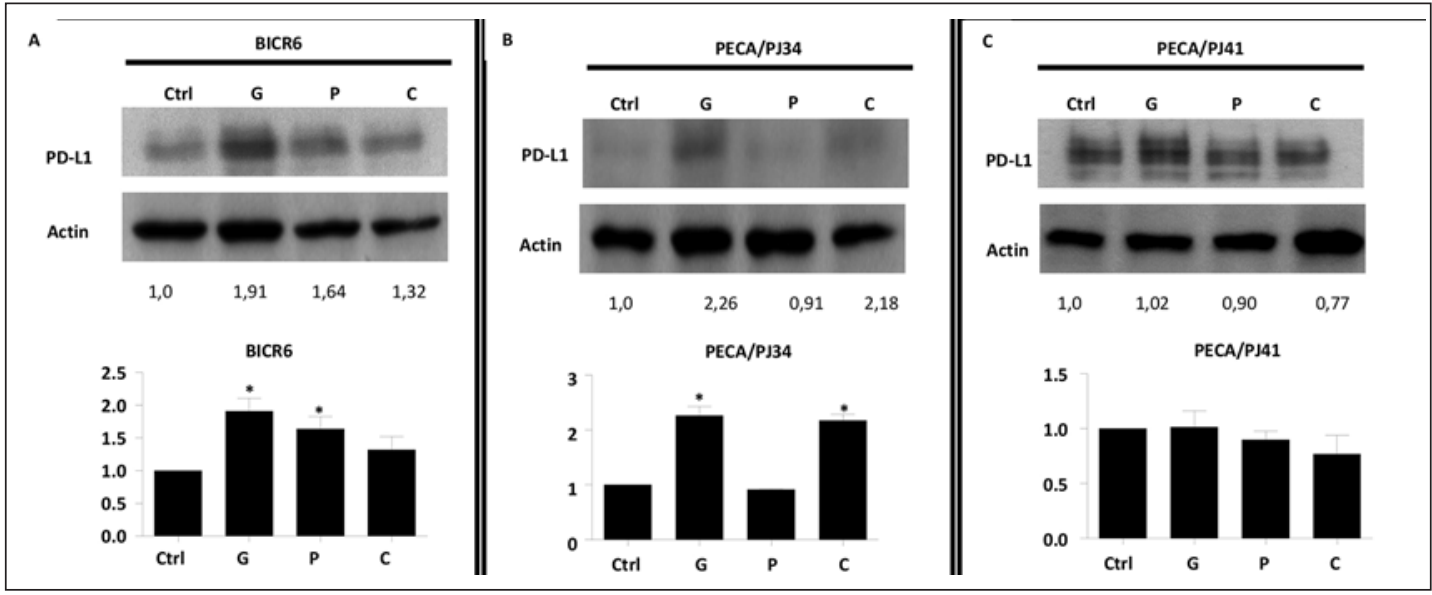

Fig. 3. A, B Significant induction in the expression of PDL-1 in BICR6 cells exposed to gemcitabine and paclitaxel and in PECA/PJ34 cells exposed to gemcitabine and cisplatin. C PDL-1 expression was not enhanced in PECA/PJ41 cells exposed to paclitaxel, gemcitabine, or cisplatin. Complete cellular extracts were prepared and analyzed by immunoblot for PDL-1 expression, using standard procedures. The ratio is a quantification of PDL-1 over actin. Three independent experiments are analyzed. ${ }^{*} p<0.05$ compared to control (Student's $t$ test).

Table 1. In vitro cytotoxicity of cisplatin, gemcitabine, and paclitaxel in human HNSCC cancer cell lines

\begin{tabular}{llll}
\hline & IC50 gemcitabine, $\mathrm{mg} / \mathrm{L}$ & IC50 paclitaxel, $\mathrm{mg} / \mathrm{L}$ & IC50 cisplatin, mg/L \\
\hline BICR6 & 0.0036 & 0.0086 & 2.8930 \\
PECA/PJ41 & 0.0176 & 0.0838 & 3.2710 \\
PECA/PJ34 & 0.2855 & 0.0925 & 6.7040 \\
\hline $\begin{array}{l}\text { Concentration used in patients } \\
\text { (ratio vs. in vitro IC50) }\end{array}$ & $200(700-55,000)$ & $20(220-2,500)$ & $20(3-7)$ \\
\hline
\end{tabular}

Exponentially growing cells were treated with the indicated drugs for $8 \mathrm{~h}$, then postincubated in drug-free culture medium for $48 \mathrm{~h}$ before fixation by ethanol and staining by Crystal Violet. After washing, cell-fixed dye was eluted in a dodecyl sulfate solution and optical densitometry was read at $590 \mathrm{~nm}$. Results are expressed as percent of control untreated cells. Half maximal inhibitory concentrations (IC50) were calculated by the PRISM software.

In vitro Cytotoxicity and PDL-1 Expression after Exposure of Human HNSCC Cell Lines to Platinum Salts, Gemcitabine, and Paclitaxel

The cytotoxicity of the drugs used for ITC in the 2 patients was evaluated in vitro using the human HNSCC cell lines PECA/PJ34, PECA/PJ41, and BICR6 (Table 1). These cell lines are HPV negative like the majority of tobacco-induced HNSCC observed in France. Carboplatin was less active than cisplatin, and the IC50 could not be determined (not shown). The ratio between the concentration used in ITC and the IC50 was lower for cisplatin than for gemcitabine and paclitaxel. PDL-1 expression was enhanced in the BICR6 cell line exposed to gemcitabine and paclitaxel (Fig. 3A) and in the PECA/PJ34 cell line exposed to gemcitabine and cisplatin (Fig. 3B). No increase in expression of PDL-1 was found in PECA/PJ41 cells (Fig. 3C). 


\section{Discussion}

To our knowledge, this is the first report of durable complete responses after treatment combining ITC with IV nivolumab in 2 heavily pretreated patients with refractory HNSCC cancer. The immune checkpoint inhibitor alone was previously inefficient in both patients. An abscopal-like effect was seen in the first patient in whom a voluminous distant axillary node was cleared after the first nivolumab re-administration combined with ITC in the locally recurrent tumor. The abscopal-like effect was only partial in the second patient. Although the tumor perfusion was nonhomogeneous, as demonstrated using Patent Blue dye (Fig. 2A), the whole tumor regressed within 2 months with dry necrosis. However, a distal mediastinal node increased in size despite nivolumab continuation and required a hypofractionated radiotherapy to obtain a metabolic response on the 18-FDG-PET scan. The complete and sustained response in both patients suggests that ITC led to a resensitization to nivolumab.

ITC has never been extensively investigated even in locoregional recurrent tumors. ITC has theoretical advantages compared to intravenous application: higher intratumoral drug concentration could overcome most of the mechanisms of chemoresistance while decreasing systemic toxicity [6]. However, the limitations of ITC are the rapid drug clearance from the tumor bed, the nonhomogeneous drug distribution, and local sides effects, such as necrosis of peritumoral tissues for some drugs, such as cisplatin, at high concentrations. Some trials showed the feasibility of ITC in locally recurrent HNSCC with a response rate of $27-50 \%$. The most frequent adverse effects were pain in $24-80 \%$ of cases, ulceration, necrosis, and edema in $53-87.4 \%$ of cases. Improvements and standardization of the technique would be necessary to define the best drugs, the optimal injection technique, treatment periodicity, and indications. We have chosen here an intratumoral infusion over a 24-h period to increase the drug persistence in the tumor (time effect in addition to the concentration effect). We hoped that the slow infusion time could increase the drug diffusion throughout the tumor bed. Platinum derivatives were chosen as core due to our experience with these compounds in ITC of recurrent HNSCC [7]. In a parallel in vitro work, we showed that cisplatin was more active than carboplatin against 3 HNSCC cell lines. Our observations show that $20 \mathrm{mg} / \mathrm{L}$ cisplatin can be safely used on head and neck tumors without normal tissue necrosis surrounding the tumor. Neither gemcitabine nor paclitaxel were necrotizing cytotoxic agents on normal tissues, and they were used here at concentrations of 200 and $20 \mathrm{mg} / \mathrm{L}$, respectively. These concentrations were far above those necessary to kill half the cells in vitro (Table 1). Moreover, our clinical observations are suggestive of an immunological effect of ITC and a resensitization to nivolumab. Immunogenic cell death (ICD) is triggered by some chemotherapeutic agents, such as anthracyclines and oxaliplatin, as well as radiation therapy [8]. The mechanism of ICD relies at least partially on the release of damage-associated molecular pattern (DAMP) molecules (calreticulin plasma-membrane translocation, ATP secretion, and/or HMGB1 release) from dying tumor cells. These molecules stimulate the recruitment of dendritic cells into the tumor bed, the uptake and processing of tumor antigens, and the optimal antigen presentation to T cells. Primed cytotoxic T lymphocytes elicit a direct cytotoxic response and kill the remaining tumor cells. This pathway activates the immune system against cancer and determines the long-term success of the checkpoint inhibitor [8,9]. The beneficial cooperation between conventional IV chemotherapy and immune checkpoint inhibitors has recently been demonstrated as a first-line treatment of recurrent HNSCC [10]. We show here 2 observations suggesting a cooperation between ITC and immunotherapy by immune checkpoint inhibitors at a later stage of the disease. We underline the potential role of gemcitabine as a component of ITC. Gemcitabine is associated with apoptosis leading to tumor antigen presentation [11], taxanes and platinum salts are associated with immune modulatory effects by increasing PDL-1 expression, which also have a key role in the sensi-

\section{Karger's}


tivity to immune checkpoint inhibitors [12]. These data are concordant with our in vitro studies where PDL-1 expression was enhanced by gemcitabine, paclitaxel, and/or cisplatin in 2 out of 3 HNSCC cell lines. Resensitization to immunotherapy after ITC that we observed suggests that local treatment may locally trigger ICD, it may educate the immune system which, in turn, will act on lesions that are distant from the initial area. It may result in an "abscopal-like" effect leading to a systemic effect from a treatment that is nevertheless localized.

\section{Conclusion}

To our knowledge, these are the first 2 cases of resensitization of locally recurrent HNSCC to the checkpoint inhibitor nivolumab after ITC. These results encouraged us to plan a phase II study assessing the tolerance and efficacy of cisplatin, gemcitabine, and paclitaxel as 24-h intratumoral infusions in combination with nivolumab in this usually hopeless situation.

\section{Acknowledgment}

We would like to thank Zuzana Saidak for English language correction and Christine Hee for technical assistance in intratumoral chemotherapy.

\section{Statement of Ethics}

We have reported these cases in compliance with the Declaration of Helsinki. Informed consent was obtained from both patients before the intratumoral treatment and for the publication of the clinical data.

\section{Conflict of Interest Statement}

The authors have no conflicts of interest to declare.

\section{Funding Sources}

The authors want to thank the "Comité de la Somme de la Ligue Nationale contre le Cancer" for funding the experimental part of the paper.

\section{Author Contributions}

Bruno Chauffert, Aline Houessinon, and Aurélie Moreira drafted the manuscript and contributed to the interpretation of the clinical results. Marine Schoonaker, Chloé Sauzay, and Antoine Galmiche designed and interpreted the experimental results. Jérèmie Bettoni, Aurélie Biet, Alexandre Coutte, Claude Krzisch, Aline Houessinon, and Bruno Chauffert treated the patients. All authors have read and approved the final manuscript.

\section{Karger's}




\section{References}

1 Gatta G, Botta L, Sánchez MJ, Anderson LA, Pierannunzio D, Licitra L. Prognoses and improvement for head and neck cancers diagnosed in Europe in early 2000s: The EUROCARE-5 Population-Based Study. Eur J Cancer. 2015 Oct;51(15):2130-43.

2 Seiwert TY, Cohen EE. State-of-the-art management of locally advanced head and neck cancer. Br J Cancer. 2005 Apr;92(8):1341-8.

3 Grégoire V, Lefebvre JL, Licitra L, Felip E; EHNS-ESMO-ESTRO Guidelines Working Group. Squamous cell carcinoma of the head and neck: EHNS-ESMO-ESTRO Clinical Practice Guidelines for diagnosis, treatment and follow-up. Ann Oncol. 2010 May;21(Suppl 5):v184-6.

4 Vermorken JB, Mesia R, Rivera F, Remenar E, Kawecki A, Rottey S, et al . Platinum-based chemotherapy plus cetuximab in head and neck cancer. N Engl J Med. 2008 Sep;359(11):1116-27.

5 Ferris RL, Blumenschein G Jr, Fayette J, Guigay J, Colevas AD, Licitra L, et al . Nivolumab for recurrent squamouscell carcinoma of the head and neck. N Engl J Med. 2016 Nov;375(19):1856-67.

6 Duvillard C, Polycarpe E, Romanet P, Chauffert B. [Intratumoral chemotherapy: experimental data and applications to head and neck tumors]. Ann Otolaryngol Chir Cervicofac. 2007;124(2):53-60.

7 Duvillard C, Romanet P, Cosmidis A, Beaudouin N, Chauffert B. Phase 2 study of intratumoral cisplatin and epinephrine treatment for locally recurrent head and neck tumors. Ann Otol Rhinol Laryngol. 2004;113(3 Pt 1):229-33.

8 Vanmeerbeek I, Sprooten J, De Ruysscher D, Tejpar S, Vandenberghe P, Fucikova J, et al . Trial watch: chemotherapy-induced immunogenic cell death in immuno-oncology. Oncoimmunology. 2020 Jan; 9(1):1703449.

9 Zitvogel L, Kepp O, Kroemer G. Immune parameters affecting the efficacy of chemotherapeutic regimens. Nat Rev Clin Oncol. 2011 Mar;8(3):151-60.

10 Burtness B, Harrington KJ, Greil R, Soulières D, Tahara M, de Castro G Jr, et al . Pembrolizumab alone or with chemotherapy versus cetuximab with chemotherapy for recurrent or metastatic squamous cell carcinoma of the head and neck (KEYNOTE-048): a randomised, open-label, phase 3 study. Lancet. 2019 Nov;394(10212): 1915-28.

11 Wargo JA, Reuben A, Cooper ZA, Oh KS, Sullivan RJ. Immune effects of chemotherapy, radiation, and targeted therapy and opportunities for combination with immunotherapy. Semin Oncol. 2015 Aug;42(4):601-16.

12 Leduc C, Adam J, Louvet E, Sourisseau T, Dorvault N, Bernard M, et al . TPF induction chemotherapy increases PD-L1 expression in tumour cells and immune cells in head and neck squamous cell carcinoma. ESMO Open. 2018 Jan;3(1):e000257. 